\title{
PREVALENCIA DE DEPRESIÓN Y FACTORES ASOCIADOS EN HOMBRES
}

\author{
Constanza Londoño Pérez ${ }^{1 *}$, Manuel González Rodríguez² \\ ${ }^{1}$ Universidad Católica de Colombia, ${ }^{2}$ Universidad de la Laguna-España
}

Recibido, septiembre 11/2015

Concepto evaluación, noviembre $5 / 2015$

Aceptado, noviembre 20/2015
Referencia: Londoño, C. \& González Rodríguez, M. (2016). Prevalencia de depresión y factores asociados en hombres. Acta Colombiana de Psicología, 19(2), 315-329. DOI: 10.14718/ ACP.2016.19.2.13

Resumen

El objetivo del presente estudio fue evaluar la prevalencia de depresión, detectar el riesgo suicida e identificar los factores sociodemográficos y personales asociados a este trastorno. La muestra no aleatorizada estuvo conformada por 1525 hombres colombianos con edades entre 18 y 83 años procedentes de 22 departamentos y de distintos niveles educativos. Para evaluar la depresión se usó el Cuestionario de Depresión para Hombres (Álvarez y Londoño, 2012); para evaluar la comorbilidad con ansiedad se usó la Escala de Ansiedad HADS (Zigmond y Snaith, 1983) y el IMAFE (Lara, 1991); y para recolectar información acerca de los factores personales y sociodemográficos se usó una ficha de registro. Se analizaron los datos para calcular la prevalencia de corte, el riesgo suicida, la comorbilidad a través del uso del paquete estadístico SPSS. Se concluye que la prevalencia real reportada y el riesgo suicida en la población estudiada son más altos que los detectados usando un instrumento no sensible al género. Palabras clave: Depresión, hombres, riesgo suicida, prevalencia.

\section{PREVALENCE OF DEPRESSION AND ASSOCIATED FACTORS IN MEN}

Abstract

This epidemiologic study aimed to evaluate the prevalence of depression, the suicide risk and the demographic and personal factors associated with depression severity in men. The non-randomized sample of participants was formed by 1525 Colombian men aged 18 to 83 years old, from 22 departments and different educational levels. The instruments used to evaluate the above factors were the Men's Depression Questionnaire (Alvarez and Londoño, 2012), the Anxiety Scale HADS (Zigmond y Snaith, 1983) and the IMAFE (Lara 1991), and in order to collect data about personal and socio-demographic factors, a registration card was used. Data were analyzed to calculate the prevalence, suicide risk and comorbidity with anxiety through the use of SPSS. It was concluded that the prevalence of depression and suicide risk in the population object of study is higher than the one identified in previous studies when a non- gender sensitive questionnaire was used.

Keys words: Depression, men, suicide risk, prevalence.

\section{PREVALÊNCIA DE DEPRESSÄO E FATORES ASSOCIADOS EM HOMENS}

Resumo

O objetivo deste estudo foi avaliar a prevalência de depressão, detectar o risco suicida e identificar os fatores sociodemográficos e pessoais associados com esse transtorno. A amostra não aleatorizada foi conformada por 1525 homens colombianos com idade entre 18 e 83 anos, procedentes de 22 estados e de diferentes níveis de escolaridade. Para avaliar a depressão, foi utilizado o Teste de Depressão para Homens (Álvarez e Londoño, 2012); para avaliar a comorbilidade com ansiedade, usou-se a Escala Hospitalar de Ansiedade e Depressão (Hads - Zigmond e Snaith, 1983) e o Inventário de Masculinidade e Feminilidade (Imafe - Lara, 1991); para coletar informação sobre os fatores pessoais e sociodemográficos, empregou-se um formulário de registro. Analisaram-se os dados para calcular a prevalência de corte, o risco suicida, a comorbilidade por meio do uso do SPSS. Concluise que a prevalência real relatada e o risco de suicídio na população estudada são mais altos do que os detectados usando um instrumento não sensível ao gênero.

Palavras-chave: depressão, homens, risco de suicídio, prevalência.

\footnotetext{
* Avenida caracas \#46-22 Piso 1 Postgrados Psicología. +0513277300 ext. 5071, clondono@ucatolica.edu.co
} 


\section{INTRODUCCIÓN}

Cuando se usa el término depresión, se hace referencia a un amplio espectro de trastornos que comparten ciertas características, que varían en sus causas, intensidad, sintomatología, cronicidad y curso, e incluso en el comportamiento epidemiológico. Es decir, que puede abordarse tanto desde la perspectiva de los síntomas como de los síndromes que incluye el síntoma anterior sumado a otros concomitantes, con una cierta gravedad y cronicidad determinadas; o desde la clasificación nosológica como trastorno mental que se expresa a través de un grupo de síntomas característicos que definen la existencia particular del trastorno (Caballo, 2006).

Las distintas formas de depresión han sido englobadas en el DSM-V (American Psychological Asociation, APA, 2013) y el Sistema de Clasificación Estadística Internacional de Enfermedades y Problemas Relacionados con la Salud [CIE-10] [Organización Panamericana de la Salud [OPS], 2012] en un grupo denominado trastornos del estado de ánimo, que se caracterizan porque la persona presenta estado de ánimo decaído y un grupo de alteraciones emocionales, cognitivas, conductuales y fisiológicas relacionadas con la condición diagnóstica principal (Vásquez, Muñoz y Becoña, 2000). Incluso se habla de una cierta ausencia de estados afectivos positivos en las personas, que se expresan en la pérdida de interés cognitivo y comportamental en las actividades de la vida cotidiana (Gotlib y Hammen, 2012).

En los trastornos del estado de ánimo ocurren oscilaciones anormales del estado de ánimo cuya intensidad, duración, asociación a estímulos e impacto sobre el desarrollo de la vida normal de la persona son notables (Friedman y Thase, 1995), de manera que se habla de la existencia de un estado melancólico anormal debido a que este no depende exclusivamente de la ocurrencia de eventos que podrían ser considerados provocadores y su duración supera los tiempos normales de reacción de tristeza de las personas; estado además asociado a síntomas emocionales, cognitivos, físicos y conductuales.

\section{Epidemiología de la depresión}

En el mundo, aproximadamente 350 millones de personas sufren de depresión y se espera que esta cifra aumente, pues se ha previsto que en 2030 la depresión será la primera causa de morbilidad mundial [Organización Mundial de la Salud (OMS), 2012], y que su impacto negativo en la productividad, en el aumento de la accidentalidad y de los costos en salud también crecerán (Córdoba, Fuentes y Ruíz, 2011).
Según la Federación Mundial de Salud Mental (FMSM, 2012), la depresión ocupa el tercer puesto en la carga global de salud mental en el mundo, y Colombia se encuentra en el quinto lugar entre los países que más registraron trastornos del estado de ánimo; pero solo países como México y Brasil han adelantado estudios censales periódicos en personas mayores de 18 años (Gómez-Restrepo et al., 2004). En México se realizó la Entrevista Nacional de Comorbilidad con tres preguntas de cribado de salud mental en una muestra aleatorizada y estratificada de 5782 personas entre 18 y 65 años; se encontró que la cohorte de población más vulnerable estaba entre 45 y 54 años, y que la prevalencia de depresión en el último año era del $8 \%$, y, a lo largo de la vida, de 9.8\% (Rafful, Medina-Mora, Borges, Benjet y Orozco, 2012).

En Colombia, en 2003 se hizo la Encuesta Nacional de Salud Mental con 4544 adultos entre 18 y 65 años en 60 municipios del país; el 14.9\% refirió haber tenido síntomas de depresión a lo largo de la vida, con distribución irregular en el territorio nacional; en Bogotá se registró el $21.1 \%$ de los casos, en la región central el $15.5 \%$ y en la zona oriental el 8.1\% [Ministerio de la Protección Social Colombia (MPSC, 2003)]. En este mismo estudio, realizado en 2015 por la misma entidad, el 5.7\% de los hombres encuestados presentaban ideación suicida y las mujeres de $7.4 \%$, sin que se publiquen aún datos específicos sobre los distintos trastornos.

En promedio, esas personas tendrán cinco eventos de depresión separados en toda su vida y al menos el $6 \%$ de los casos desembocará en un intento de suicidio (Gobierno de Canarias, 2010). Así mismo, el 15\% de los pacientes reúnen criterios de cronicidad, ya que reportan depresión continua por más de 2 años, asociada a peor ajuste social, alto deterioro funcional e incremento en el uso de servicios sanitarios, y solo el $1 \%$ de la población reporta un episodio depresivo que se prolonga por más de 5 años (Conradi, Ormel y De Jonge, 2011).

En el Estudio de Salud Mental de 2003 los hombres reportaron prevalencias globales más altas en todos los trastornos; sin embargo, y a pesar de la dinámica social, no existe un sistema nacional de estadísticas que permita hacer un verdadero seguimiento a los alcances de los problemas de salud mental en este país.

En Colombia, y en el resto del mundo, la mayoría de reportes de depresión provienen de mujeres que acuden a centros asistenciales en busca de ayuda profesional, y estos centros a su vez reportan a las entidades gubernamentales las estadísticas acerca de los motivos de consulta, de tal modo que las estadísticas referidas a la prevalencia de depresión sin duda son predominantemente femeninas. 
En estudios anteriores se ha evidenciado que las mujeres deprimidas son más proclives que los hombres deprimidos a usar los servicios sanitarios o a solicitar soporte social, pues la población masculina tiene dificultad para reconocer los síntomas de depresión, y si los perciben, prefieren evitarlos consumiendo drogas o con mecanismos extremos de escape como el suicidio. Cabe notar que los hombres se suicidan más que las mujeres y que al menos en la mitad de los casos lo hacen a causa de una depresión (Haeffel et al., 2007; Instituto Nacional de Medicina Legal y Forense, 2013; Merikangas et al., 2011; Mittendorfer-Rutz, 2006; Troister y Holden, 2010).

En los estudios epidemiológicos antes mencionados se replican las limitaciones en la evaluación que se han presentado a lo largo de la historia del estudio de la depresión; sin duda, el diagnóstico certero realizado a través de instrumentos es un reto, pues no son pocos los obstáculos por sortear para lograr medidas precisas. Entre estos se encuentran el solapamiento de síntomas y la no identificación de síntomas centrales claramente diferenciales que provocan alta convergencia entre las escalas usadas para medir dos entidades diagnósticas consideradas diferentes, como, por ejemplo, depresión $\mathrm{y}$ ansiedad. Muchos de los instrumentos usados fueron diseñados hace largo tiempo y algunos aún no han sido reformados o actualizados; además, concentran su atención en síntomas cognitivos minimizando el valor diagnóstico de los síntomas conductuales, somáticos y emocionales; se busca la brevedad en los cuestionarios para favorecer la reducción de costos, pero se afecta la capacidad discriminante de los instrumentos al aumentar el tipo de error de medición aceptable, y se usan medidas diagnósticas para hacer tamizajes o detección temprana.

La dificultad en la identificación temprana y el diagnóstico de depresión en hombres es aún más crítica debido a la ineficiencia de los sistemas de detección de casos, a las fallas en la inclusión de muestras masculinas en los estudios tipo y al uso de instrumentos con sesgo, que dificultan el desarrollo de tratamientos costo-efectivos dirigidos específicamente a los hombres. Adicionalmente, los sistemas de salud prevén poco tiempo de consulta, lo cual reduce la oportunidad que tienen de los profesionales de la salud de diagnosticar adecuadamente los casos de depresión; esto se suma al hecho de que los médicos generales evitan de forma implícita dictaminar enfermedad mental, pues la aplicación de los protocolos de atención definida para estos trastornos incrementa el compromiso económico de las entidades prestadoras de servicios sanitarios.

\section{Factores asociados}

El estudio de los orígenes de la depresión ha permitido conocer que es multicausal, y que en su aparición intervienen factores biológicos, psicológicos, sociales y ambientales. Es así como se puede decir que tanto el fenotipo intermediador (hiperreactividad genética al estrés) como las experiencias tempranas vividas por el individuo actúan como factores predisponentes de la depresión, ya que modulan la respuesta más o menos aguda de estrés desencadenada por los acontecimientos estresantes, la cual, a su vez, es considerada precursora de la depresión (Caspi, Hariri, Holmes, Uher y Moffitt, 2010; Karg, Burmesister, Shedden y Sen, 2011)

Así mismo, el ambiente social inmediato actúa como facilitador de la aparición y agudización de la depresión, más que como desencadenante. Es decir, que eventos sociales adversos, considerados parte del ambiente social, facilitan la aparición de una respuesta desproporcionada a la magnitud del evento, y que el evento en sí mismo solo tiene un cierto nivel potencial dañino, dependiente de las condiciones socioeconómicas, el grado de soporte social, y los roles de género ejercidos, entre otras (Mejía Castillo, 2011; Sandín, 2003).

Diversos estudios han mostrado que las personas que viven con limitaciones económicas, además de que ven reducida su posibilidad de acceder a opciones educativas, de vivienda, trabajo, de salud y recreación (Moore y Hadjiyannakis, 2002; [Organización Panamericana de la Salud (OPS), 2012] tienden a reportar problemas de salud mental; parece que la situación de pobreza actúa como estresor crónico y afecta la capacidad de afrontar el estrés; incluso el apoyo social recibido se ve disminuido debido a la necesidad de suplir exigencias de supervivencia de cada uno de los miembros del grupo familiar casi de forma individual (Almeida et al., 2012; Juárez y Guerra, 2011; Lund et al., 2010; Huurre, Eerola, Rahkonen y Does, 2006; Palomar y Cienfuegos, 2007).

Es suficientemente conocido que la historia familiar de trastornos mentales incrementa el riesgo de padecer depresión, ya sea debido a la expresión de ciertos factores heredados o al hecho de que las personas con depresión reportan más problemas parentales, ofrecen bajo apoyo a los miembros de la familia e incumplen su rol familiar de soporte social (Heponiemi et al., 2006; Sander y MacCarthy, 2005; Vallejo Casarín, Osorno Murguia y Mazadiego Infante, 2008).

Diferentes investigaciones ponen de manifiesto que un número importante de experiencias estresantes como: 
problemas de salud, maltrato, violencia escolar y disfunción familiar se asocian a la aparición de la depresión (Dokin et al., 2013; Drydakis, 2013; Fellinger, Holzinger, Sattel, Laucht y Goldberg, 2009; Ibarra-Alcantar, Ortiz-Guzmán, Graciano-Morales y Jiménez-Genchi, 2010; Pawlby, Hay, Sharp, Waters y O'keane, 2009); condición que se confirma si se considera que biomarcadores como el cortisol han sido ubicados en la sangre de un buen número de pacientes con algún tipo de depresión (Adam et al., 2010; VrshekSchallhorn et al., 2012). Esto quiere decir que la exposición a eventos agudos o crónicos de estrés se asocia al incremento de la vulnerabilidad en salud mental, lo cual a su vez agudiza las condiciones crónicas de salud (Almeida et al., 2011; Instituto Nacional de Salud Mental, 2013) y esto potencia la misma depresión.

A pesar de los avances en el conocimiento de los diversos factores que potencian el riesgo de padecer un trastorno del estado de ánimo, es difícil calcular y predecir el riesgo real de un persona; más aún en un grupo poblacional específico. Existen múltiples factores culturales, sociales, estructurales y psicológicos que han sido asociados a la aparición, curso y posibilidades de recuperación de los distintos trastornos del estado de ánimo, factores que interactúan con frecuencia en un medio complejo como lo es la sociedad actual y en especial un país como Colombia.

En los estudios epidemiológicos, en general usan medidas como el BDI, la escala Hamilton y otros instrumentos construidos con muestras eminentemente femeninas; ejemplo de ello es el Estudio Nacional de Salud Mental en Colombia que usó el BDI I, los estudios periódicos que hace la OMS, la Encuesta Mundial de Salud Mental y otros estudios internacionales (MPSC, 2003; FMSM, 2012; OMS, 2011; OPS, 2005), cuya sensibilidad a los cuadros masculinos de depresión, en especial en el caso del BDI en ambas versiones, es reducida. Ya Chang et al. (2008) habían encontrado serias diferencias en las estadísticas epidemiológicas en la misma población, dependientes de las escalas usadas. Cabe aclarar que no se espera que al ajustar las medidas el perfil epidemiológico dé un giro radical, pero sí que se hagan más sensibles y discriminativas ante la variable género y la especificidad de la expresión de los trastornos del estado de ánimo dependiente del grado de masculinidad/feminidad expresado por cada persona y que se conduzcan estudios de corte censal con muestras eminentemente masculinas, para que exista mayor confianza en las cifras epidemiológicas disponibles. Cabe preguntarse entonces cuál es la prevalencia de depresión masculina y cuáles son los factores psicosociales y ambientales asociados.

\section{MÉTODO}

El presente es un estudio de tipo epidemiológico realizado en población masculina colombiana,

\section{Participantes}

La muestra de participación voluntaria, estratificada por edad para lograr representatividad de jóvenes, adultos y adultos mayores; y por lugar de procedencia, para garantizar participación homogénea de la mayor cantidad de departamentos de Colombia. La muestra calculada con el software gPower estaba conformada por 2200 hombres que fueron abordados en grupos de 15 a 35 personas en empresas, instituciones de educación (media vocacional y superior), entidades gubernamentales y no gubernamentales contactadas a través de cartas de solicitud, ya que fueron identificadas previamente porque estaban conformadas por hombres que cumplían los criterios de edad y procedencia. De esa muestra inicial, 1525 hombres aceptaron participar, de los cuales se eliminaron 115 casos debido a la no firma del consentimiento informado, a sesgos o a omisión de respuestas.

Entre los criterios de inclusión del estudio estaban ser hombre colombiano, residir en el territorio nacional, ser mayor de 18 años y saber leer y escribir, además de acceder voluntariamente a participar en la encuesta.

Las edades fueron de 18 a 83 años, con una media de edad de 26.59 años (DT = 9.45) principalmente jóvenes $(40.2 \%, 22$ a 35 años) y adolescentes mayores $(40.2 \%, 18$ a 21 años) que vivían principalmente acompañados de su familia (91.1\%); con distintos niveles educativos, entre ellos universitario (40\%), bachillerato (34.2\%) y técnico (14.4\%); de nivel socioeconómico principalmente medio bajo (estratos 2 y 3 , con $27.5 \%$ y $46.9 \%$, respectivamente) . Provenientes de aproximadamente 95 municipios ubicados en 22 departamentos localizados en las zonas de más densa población del territorio nacional (véase Tabla 1). 
Tabla 1.

Descripción de las condiciones sociodemográficas de los 1413 hombres participantes en el estudio

\begin{tabular}{|c|c|c|c|c|c|}
\hline Variable & $\mathrm{f}$ & $\%$ & Variable & $\mathrm{f}$ & $\%$ \\
\hline Nivel educativo & & & Procedencia & & \\
\hline Primaria & 45 & 3.2 & Cundinamarca & 308 & 21.8 \\
\hline Bachillerato & 480 & 34.2 & Santander & 119 & 8.4 \\
\hline Técnico & 203 & 14.4 & Tolima & 95 & 3.7 \\
\hline Universitario & 570 & 40.3 & Valle & 89 & 6.3 \\
\hline Postgrados & 115 & 8.1 & Boyacá & 154 & 10.9 \\
\hline Estrato & & & Quindio & 22 & 1.6 \\
\hline 1 & 99 & 7 & Arauca & 1 & 0.1 \\
\hline 2 & 389 & 27.5 & Antioquia & 100 & 7.1 \\
\hline 3 & 663 & 46.9 & Cauca & 5 & 0.3 \\
\hline 4 & 217 & 15.4 & Nariño & 23 & 1.6 \\
\hline 5 & 37 & 2.6 & Huila & 71 & 5.0 \\
\hline 6 & 8 & 0.6 & Casanare & 10 & 0.7 \\
\hline Ocupación & & & Magdalena & 81 & 5.7 \\
\hline Estudia & 340 & 24.1 & Meta & 12 & 0.8 \\
\hline Trabaja & 466 & 33.0 & Bolivar & 21 & 1.5 \\
\hline Estudia/trabaja & 304 & 21.5 & Cesar & 146 & 10.3 \\
\hline Otro & 183 & 13.0 & Atlántico & 20 & 1.4 \\
\hline Desempleado & 120 & 8.4 & Guajira & 51 & 3.6 \\
\hline Vive con & & & Caldas & 57 & 4.0 \\
\hline Familia & 1285 & 91.1 & San Andrés & 1 & 0.1 \\
\hline Amigos & 12 & 0.8 & Córdoba & 26 & 1.8 \\
\hline Otros & 102 & 7.2 & & & \\
\hline Solo & 12 & 0.8 & & & \\
\hline \multicolumn{6}{|l|}{ Grupos de edad } \\
\hline 18 a 21 & 568 & 40.2 & & & \\
\hline 22 a 35 & 622 & 44.0 & & & \\
\hline 36 a 50 & 180 & 2.7 & & & \\
\hline 51 o más & 43 & 3.0 & & & \\
\hline
\end{tabular}

Notas: f: Frecuencia; \%: Porcentaje.

\section{Instrumentos}

Ficha de información sociodemográfica

Esta es una ficha de registro que recoge información como género, edad, nivel educativo, procedencia, ocupación y nivel de satisfacción con la actividad laboral realizada, estrato socioeconómico, conformación y funcionamiento familiar (APGAR, Adaptability Partnertship Growth Affection Resolve, Smilkstein, 1978), historia y antecedentes psicopatológicos familiares.
El Cuestionario de APGAR Familiar

(Adaptability Partnertship Growth Affection Resolve) evalúa el nivel de funcionamiento familiar y fue validado para población colombiana por Forero, Avendaño, Duarte y Campo-Arias (2006). La escala está compuesta por dos factores, disfunción familiar y soporte familiar. La consistencia interna global es de 0,79 , pero no se ha calculado la consistencia de cada dimensión. 
Cuestionario de Depresión en Hombres (CDH)

El cuestionario fue diseñado por Álvarez y Londoño (2012) y mide seis dimensiones: autoimagen negativa, ideación suicida, desesperanza, pobre imagen social, evitación y afecto negativo cuenta con un nivel de fiabilidad elevado de 0.95 y 0.96 en los índices calculados desde la Teoría Clásica de los Test y desde la Teoría de Respuesta al Ítem, respectivamente, y un índice de separación adecuado de 4,19, que indica un ajuste adecuado.

\section{Escala de Ansiedad-Depresión Hospitalaria}

(HADS - Hospital Anxiety and Depression Scale): Diseñada por Zigmond y Snaith (1983); es utilizada en el cribado de trastornos de depresión y ansiedad, ya que proporciona de manera rápida una puntuación en ambos problemas psicológicos. Validada por Rico, Restrepo y Molina (2005), para población colombiana. La Escala de Ansiedad está compuesta por 7 ítems con opciones de respuesta en escala Likert de 4 opciones que van de 0 a 3 . El HADS cuenta con un adecuado nivel de confiabilidad reflejado en el alfa de Cronbach reportado de 0.85 para toda la escala; menor en las escalas específicas pero adecuado, 0.76 para la escala de ansiedad y 0.77 para la de depresión.

Escala de Eventos Vitales Estresantes de la OMS: Adaptada por Oliva et al. (2008) y validada en población colombiana por Gómez et al. (2012), se usa para evaluar la existencia de condiciones ambientales provocadoras de estrés y la intensidad de su efecto. Consta de ítems que describen brevemente 23 sucesos potencialmente estresantes para las personas, obteniendo una fiabilidad de 0.70 .

\section{Escala de Rol de Masculinidad Feminidad}

Desarrollada en Colombia por Lara (1991) y revalidada por Martínez, Guerrero y Rey (2012), analiza el rol social asumido por las personas; consta de 60 ítems que incluyen adjetivos descriptivos de los roles, los cuales se subdividen en cuatro factores, a saber: masculinidad, feminidad, machismo y sumisión. El factor de masculinidad incluye adjetivos que describen rasgos positivos considerados característicos de los varones; el factor feminidad incluye adjetivos que describen rasgos positivos considerados femeninos o característicos de las mujeres; el factor machismo engloba adjetivos que describen características masculinas negativas, y el factor sumisión incluye adjetivos que describen rasgos negativos de la feminidad, centrados precisamente en la sumisión. Las opciones de respuesta van de 1 a 7 en una escala Likert que ofrece diversos grados de frecuencia en los que la persona puede expresar el rasgo definido en cada adjetivo. Los valores del alfa de Cronbach de las cuatro escalas fueron 0.78 en el factor masculinidad, 0.88 en el factor feminidad, 0.82 en el factor machismo y 0.79 en el factor de sumisión.
Cuestionario de Estilos de Afrontamiento

Desarrollado por Lazarus y Folkman (1985) y validado por Rueda y Vélez-Botero (2010) para población colombiana. Está conformado por 42 ítems que evalúan el estilo de afrontamiento predominante ante eventos estresantes. Estos estilos se agrupan en 5 factores, a saber: solución de problemas, evitación, fantasía, autoculpa y búsqueda de soporte social. Las opciones de respuesta van en una escala Likert de 4 opciones. El cuestionario cuenta con una fiabilidad de 0.70 para la escala total, 0.88 para la escala de solución de problemas, 0.75 para la de búsqueda de soporte social, 0.74 para la de evitación, 0.78 para la de autoculpa y 0.85 para la de fantasía.

\section{Procedimiento}

En primer lugar, se hizo una convocatoria de estudiantes de psicología de los últimos semestres a través del Nodo de Psicología y Salud de la Red de Investigadores de Ascofapsi, quienes en un segundo momento presentaron entrevista de selección, aval de un investigador y firmaron carta de compromiso; en tercer lugar, estos auxiliares fueron entrenados y acompañados para la aplicación. Posteriormente, en cuarto lugar, se aplicaron los instrumentos según la guía. Para calcular la prevalencia de corte se usó la fórmula Prevalencia Puntual $=$ Ct número de casos existentes (prevalentes) $/ \mathrm{Nt}$ número total de individuos.

\section{RESULTADOS}

A continuación se presentan los resultados descriptivos de las medidas obtenidas durante el estudio, con especial énfasis en los resultados referidos a la depresión y a los factores asociados a la severidad de esta en hombres a través de un Modelo de Ecuaciones Estructurales.

De los hombres encuestados, el 10.9\% de la población presentaba síntomas de depresión en un nivel subclínico; el $6.9 \%$ alcanzó puntajes que sobrepasaron el punto de corte clínico (106 puntos); el 6.1\% reportó un nivel de severidad moderado y el $0.8 \%$ de depresión severa durante el último mes. Analizando por dimensiones del CDH, en imagen negativa de símismo el $7.1 \%$ presentó un nivel alto, el $18.1 \%$ un nivel moderado y el $74.6 \%$ un nivel bajo. Asimismo el $7.7 \%$ de la población presentó ideación suicida, el 12.2\% tenía ideas de muerte. El 18.9\% refirió tener pobre imagen social alta y el $51.9 \%$ moderada; por otra parte, el $18.6 \%$ reportó tener afecto negativo alto y el $34.5 \%$ un nivel moderado. Para el caso del factor desesperanza, el 1.9\% mostró alta desesperanza y el $22.7 \%$ moderada; finalmente el $14.4 \%$ presentó evitación moderada y el $4.2 \%$ alta (véase Tabla 2). 
Tabla 2.

Prevalencia de depresión en la muestra objeto de estudio y su nivel de severidad

\begin{tabular}{|c|c|c|c|c|c|}
\hline Variable & $\mathrm{N}$ & $\%$ & Variable & $\mathrm{N}$ & $\%$ \\
\hline Depresión & & & F1: Imagen negativa & & \\
\hline Sin depresión & 1161 & 82.2 & Baja & 1054 & 74.6 \\
\hline \multirow[t]{2}{*}{ Depresión clínica } & 98 & 6.9 & Moderada & 256 & 18.1 \\
\hline & & & Alta & 103 & 7.1 \\
\hline Población & & & F2: Ideación suicida & & \\
\hline Sin depresión & 1161 & 82.2 & Ausente & 1032 & 80.1 \\
\hline Depresión leve & 154 & 10.9 & Ideas de muerte & 173 & 12.2 \\
\hline \multirow[t]{2}{*}{ Depresión } & 98 & 7.9 & Ideación suicida & 108 & 7.7 \\
\hline & & & F3: Pobre imagen social & & \\
\hline Nivel de severidad & & & Bajo & 412 & 29.2 \\
\hline Depresión moderada & 88 & 6.1 & Medio & 734 & 51.9 \\
\hline \multirow[t]{13}{*}{ Depresión severa } & 10 & 0.8 & Alta & 267 & 18.9 \\
\hline & & & F4: Afecto negativo & & \\
\hline & & & Bajo & 662 & 46.9 \\
\hline & & & Medio & 488 & 34.5 \\
\hline & & & Alto & 253 & 18.6 \\
\hline & & & F5: Desesperanza & & \\
\hline & & & Baja & 1071 & 75.8 \\
\hline & & & Media & 315 & 22.3 \\
\hline & & & Alta & 27 & 1.9 \\
\hline & & & F6: Evitación & & \\
\hline & & & Baja & 1151 & 81.5 \\
\hline & & & Media & 203 & 14.4 \\
\hline & & & Alta & 59 & 4.2 \\
\hline
\end{tabular}

Notas: $\mathrm{n}: 1413$.

Del total de la población, el $12.2 \%$ tenía antecedentes familiares de depresión, el 9.9\% de consumo de sustancias psicoactivas, el $4.4 \%$ de suicidio, el $8.9 \%$ de conducta violenta y el $8.6 \%$ de comportamiento arriesgado. Las medias reportadas de funcionamiento familiar (APGAR) tenían un promedio bajo de disfunción familiar (1.12) y un promedio moderado de bajo de soporte familiar percibido (2.02).

Entre los factores culturales evaluados, se encontró que cerca del $100 \%$ de los varones encuestados informaron ser heterosexuales. En el mismo grupo de factores, se evidenció que la media más alta en la prueba de masculinidad/ feminidad fue la del factor masculinidad (4.14), seguida de la media de machismo (3.86), de la de feminidad (3.82) y la de sumisión (3.49); todas con desviaciones estándar menores a 0.85 , lo cual indica baja dispersión en los datos. En los factores ambientales, se evidenció que los hombres habían vivido en promedio cerca de 6 Eventos Vitales Estresantes (EVE) en el último año (5.56), con una media alta de impacto psicológico (7.06). Se encontró que entre los cinco EVE más reportados por los hombres estaban: logro personal notable (38.6\%), cambio en las condiciones de vida $(32.9 \%)$, vacaciones $(32.6 \%)$, navidad $(32.2 \%)$ y cambio de salud de algún familiar (31.2\%) (véase Tabla 3).

Los cinco EVE que causaron mayor impacto psicológico en promedio en los hombres encuestados fueron: logro personal notable $(\mathrm{M}=7.93)$, embarazo de su pareja $(\mathrm{M}=7.55)$, muerte de un familiar cercano $(\mathrm{M}=7.54)$, navidad $(\mathrm{M}=7.47) \mathrm{y}$ vacaciones $(\mathrm{M}=7.41)$; $\mathrm{y}$ los tres con menor impacto reportado, sin que este llegara a ser bajo, estaban: lesión o enfermedad personal $(\mathrm{M}=6.44)$, problemas sexuales $(\mathrm{M}=6.62)$ y cambio de actividades de ocio $(\mathrm{M}=6.78)$ (véase Tabla 3$)$.

En lo que se refiere a los factores psicológicos evaluados, se encontró que el puntaje medio de ansiedad era moderado $(\mathrm{M}=6.38)$, el de depresión era no clínico $(\mathrm{M}=69.11)$. En los factores del CDH, la autoimagen negativa se ubicó en el extremo más alto del nivel bajo $(\mathrm{M}=6.22)$, al igual que 
el de ideación suicida $(\mathrm{M}=5.70)$; y en los niveles medios se ubican pobre imagen social $(\mathrm{M}=9.88)$, afecto negativo $(\mathrm{M}=27.76)$, desesperanza $(\mathrm{M}=5.43)$ y evitación $(\mathrm{M}=14.12)$. Tomando en cuenta los estilos de afrontamiento, la media más alta corresponde al estilo de afrontamiento solución de problemas $(\mathrm{M}=2.42)$, seguida de búsqueda de soporte social $(\mathrm{M}=2.26)$, fantasia $(\mathrm{M}=2.17)$, autoculpa $(\mathrm{M}=2.07)$; y en último lugar, evitación ( $\mathrm{M}=1.98)$.

Los hombres encuestados presentaron medias de satisfacción moderada en todas las áreas de su vida, con la media más alta en la satisfacción con la familia $(\mathrm{M}=5.16)$ y en la satisfacción con la vida en general $(\mathrm{M}=4.90)$; $\mathrm{y}$ con las medias más bajas en la satisfacción con las condiciones económicas actuales $(\mathrm{M}=4.19)$ y con el tiempo libre $(\mathrm{M}=4.17)$ (véase Tabla 3).
En los EVE se encuentra que los cinco más frecuentemente reportados por los hombres fueron: logro personal notable (38.9\%), cambio en las condiciones de vida (32.9\%), vacaciones (32.6\%), navidad (32.2\%) y cambio en la salud de un familiar (31.2\%); y los dos de menor frecuencia fueron problemas legales $(9.5 \%)$ y leves trasgresiones de la ley (8.3\%). Mientras que los cinco que produjeron más impacto psicológico en promedio fueron el logro personal notable $(\mathrm{M}=7.9)$, embarazo de la pareja $(\mathrm{M}=7.55)$, muerte de un familiar cercano $(\mathrm{M}=7.54)$, incorporación de un nuevo miembro a la familia $(\mathrm{M}=7.51)$ y navidad $(\mathrm{M}=7.47)$; y las dos de menor impacto fueron lesión o enfermedad personal $(\mathrm{M}=6.44)$ y cambio en el número de reuniones familiares $(\mathrm{M}=6.55)$ (véase Tabla 4).

Tabla 3.

Datos descriptivos de los factores sociales, culturales, ambientales y psicológicos evaluados en la muestra

\begin{tabular}{|c|c|c|c|c|c|c|c|c|c|}
\hline Variable & $\mathrm{f}$ & \multicolumn{2}{|c|}{$\%$} & & Variable & $\mathrm{f}$ & \multicolumn{2}{|c|}{$\%$} & \\
\hline Antecedentes familiares & & & & \multicolumn{2}{|r|}{ Género } & & & & \\
\hline Suicidio & 62 & \multicolumn{2}{|c|}{4.4} & & Heterosexuales & 1356 & \multicolumn{2}{|c|}{96} & \\
\hline Consumo drogas & 133 & \multicolumn{2}{|c|}{9.4} & & Homosexuales & 39 & \multicolumn{2}{|c|}{2.7} & \\
\hline Depresión & 173 & \multicolumn{2}{|c|}{12.2} & & Bisexuales & 17 & \multicolumn{2}{|c|}{1.2} & \\
\hline Conducta violenta & 126 & \multicolumn{2}{|c|}{8.9} & & Otro & 1 & \multicolumn{2}{|c|}{01} & \\
\hline Comportamiento arriesgado & 121 & \multicolumn{2}{|c|}{8.6} & & & & & & \\
\hline Variable & mín & $\max$ & $\mathrm{M}$ & DT & Variable & mín & $\max$ & M & DT \\
\hline Ansiedad & 0 & 17 & 6.38 & 3.38 & Estilo de afrontamiento & & & & \\
\hline Depresión & 0 & 160 & 69.11 & 18.99 & Solución de problemas & 1 & 4 & 2.42 & 0.49 \\
\hline Imagen negativa de sí mismo & 0 & 16 & 6.22 & 2.36 & Soporte social & 1 & 4 & 2.26 & 0.55 \\
\hline Ideación suicida & 0 & 16 & 5.70 & 2.40 & Evitativo & 1 & 4 & 1.98 & 0.44 \\
\hline Pobre imagen social & 0 & 24 & 9.88 & 3.36 & Autoculpa & 1 & 4 & 2.07 & 0.65 \\
\hline Afecto negativo & 0 & 60 & 27.76 & 7.85 & Fantasía & 1 & 4 & 2.17 & 0.67 \\
\hline Desesperanza & 0 & 12 & 5.43 & 1.77 & & & & & \\
\hline \multirow[t]{2}{*}{ Evitación } & 0 & 32 & 14.12 & 3.96 & Masculinidad/feminidad & & & & \\
\hline & & & & & Masculinidad & 1 & 7 & 4.14 & 0.84 \\
\hline APGAR & & & & & Feminidad & 1 & 7 & 3.82 & 0.78 \\
\hline Disfunción & 0 & 8 & 1.12 & 1.30 & Machismo & 1 & 7 & 3.86 & 0.82 \\
\hline Soporte & 0 & 5 & 2.02 & 0.99 & Sumisión & 1 & 7 & 3.49 & 0.80 \\
\hline EVE & & & & & Satisfacción & & & & \\
\hline Número EVE & 0 & 22 & 5.56 & 5.02 & Vida en general & 1 & 6 & 4.90 & 1.00 \\
\hline \multirow[t]{2}{*}{ Impacto } & 6 & 9 & 7.06 & 0.72 & Trabajo & 1 & 6 & 4.46 & 1.15 \\
\hline & & & & & Familia & 1 & 6 & 5.16 & 0.96 \\
\hline \multirow[t]{6}{*}{ Uso drogas } & 0 & 10 & 2.60 & 1.45 & Pareja & 1 & 6 & 4.58 & 1.24 \\
\hline & & & & & Condiciones económicas & 1 & 6 & 4.19 & 1.16 \\
\hline & & & & & Amigos & 1 & 6 & 4.64 & 1.02 \\
\hline & & & & & Tiempo libre & 1 & 6 & 4.17 & 1.32 \\
\hline & & & & & Estudio & 1 & 6 & 4.49 & 1.21 \\
\hline & & & & & Salud & 1 & 6 & 4.84 & 1.02 \\
\hline
\end{tabular}


Tabla 4.

EVE el logro personal notable (38.9)

\begin{tabular}{|c|c|c|c|c|c|c|}
\hline Variables & $\mathrm{N}$ & $\%$ & mín & $\max$ & M & DT \\
\hline \multicolumn{7}{|l|}{ EVE } \\
\hline \multicolumn{7}{|l|}{ Impacto de los EVE } \\
\hline Muerte de familiar cercano & 371 & 26.3 & 4 & 10 & 7.54 & 1.95 \\
\hline Lesión o enfermedad personal & 317 & 22.4 & 4 & 10 & 6.44 & 1.81 \\
\hline Despido del trabajo & 149 & 10.5 & 4 & 10 & 6.88 & 1.98 \\
\hline Cambio de salud de un familiar & 441 & 31.2 & 4 & 10 & 7.02 & 1.80 \\
\hline Embarazo & 146 & 10.3 & 4 & 10 & 7.55 & 2.09 \\
\hline Dif. o problemas sexuales & 162 & 11.5 & 4 & 10 & 6.62 & 1.99 \\
\hline Incorp. nuevo miembro familia & 216 & 15.3 & 4 & 10 & 7.51 & 2.09 \\
\hline Muerte de un amigo intimo & 180 & 12.7 & 4 & 10 & 7.25 & 1.96 \\
\hline Problemas legales & 134 & 9.5 & 4 & 10 & 7.14 & 1.89 \\
\hline Logro personal notable & 549 & 38.9 & 4 & 10 & 7.93 & 1.77 \\
\hline Cambio en las condiciones de vida & 465 & 32.9 & 4 & 10 & 7.24 & 1.83 \\
\hline Cambio de residencia & 299 & 21.2 & 4 & 10 & 7.00 & 2.00 \\
\hline Cambio institución educativa/trabajo & 272 & 19.2 & 4 & 10 & 7.12 & 1.88 \\
\hline Cambio de actividad de ocio & 272 & 19.2 & 4 & 10 & 6.78 & 1.92 \\
\hline Cambio de actividad religiosa & 148 & 10.5 & 4 & 10 & 6.98 & 2.01 \\
\hline Cambio de actividades sociales & 307 & 21.7 & 4 & 10 & 6.88 & 1.89 \\
\hline Cambio de hábito de dormir & 404 & 28.6 & 4 & 10 & 7.14 & 2.08 \\
\hline Cambio número reuniones familiares & 252 & 17.8 & 4 & 10 & 6.58 & 1.88 \\
\hline Cambio de hábitos alimentarios & 379 & 26.8 & 4 & 10 & 6.95 & 1.91 \\
\hline Vacaciones & 461 & 32.6 & 4 & 10 & 7.41 & 1.95 \\
\hline Navidad & 455 & 32.2 & 4 & 10 & 7.47 & 2.01 \\
\hline Leves transgresiones de la ley & 117 & 8.3 & 4 & 10 & 6.89 & 1.7 \\
\hline
\end{tabular}

Notas: Hombres $(\mathrm{n}=1413) \mathrm{f}$.

Una vez presentados los análisis descriptivos, se procedió a hacer el análisis de factores asociados. En la Figura 1 se presenta el modelo de medición y el modelo resultante de los factores asociados a la severidad de la depresión en los hombres, en el que variables como EVE (Peso Relativo de Varianza Explicado $[\mathrm{PRVE}]=0.38$ ) la ansiedad ( $\mathrm{PRVE}=0.37$ ), los estilos de afrontamiento ( $\mathrm{PRVE}=0.38$ ), el funcionamiento familiar (PRVE=0.32) y el género (PRVE=0.16) en interacción explican el 66\% de varianza de la severidad de la depresión en hombres.

En primer lugar, como era esperable después del análisis, se encuentra un estilo de afrontamiento mixto conformado por la evitación con mayor peso de varianza explicada aportado (PRVE=0.21), seguido del aporte negativo que hace el estilo centrado en la solución de problemas (PRVE=-
0.11) y del aporte positivo del afrontamiento centrado en la auto culpa (PRVE=0.10).

En segundo lugar, están las variables (latentes) del modelo denominadas acontecimietos vitales estresantes y el funcionamiento familiar ( $\mathrm{PRVE}=0.32$ respectivamente); en la primera se muestra que el impacto psicológico aporta en mayor medida y de forma negativa ( $\mathrm{PRVE}=-0.56$ ), que el número de eventos reportados por la persona ( $\mathrm{PRVE}=0.03$ ), a la explicación de varianza de la severidad de la depresión. Respecto del funcionamiento familiar se encontró que la disfunción familiar aporta un peso relativo de varianza positivo (PRVE=0.15) y el soporte familiar aporta de forma negativa ( $\mathrm{PRVE}=-0.19)$. En cuarto lugar, se encontró que el rol de género aportaba el menor peso relativo de varianza explicada (PRVE=0.16) con la masculinidad y la sumisión 


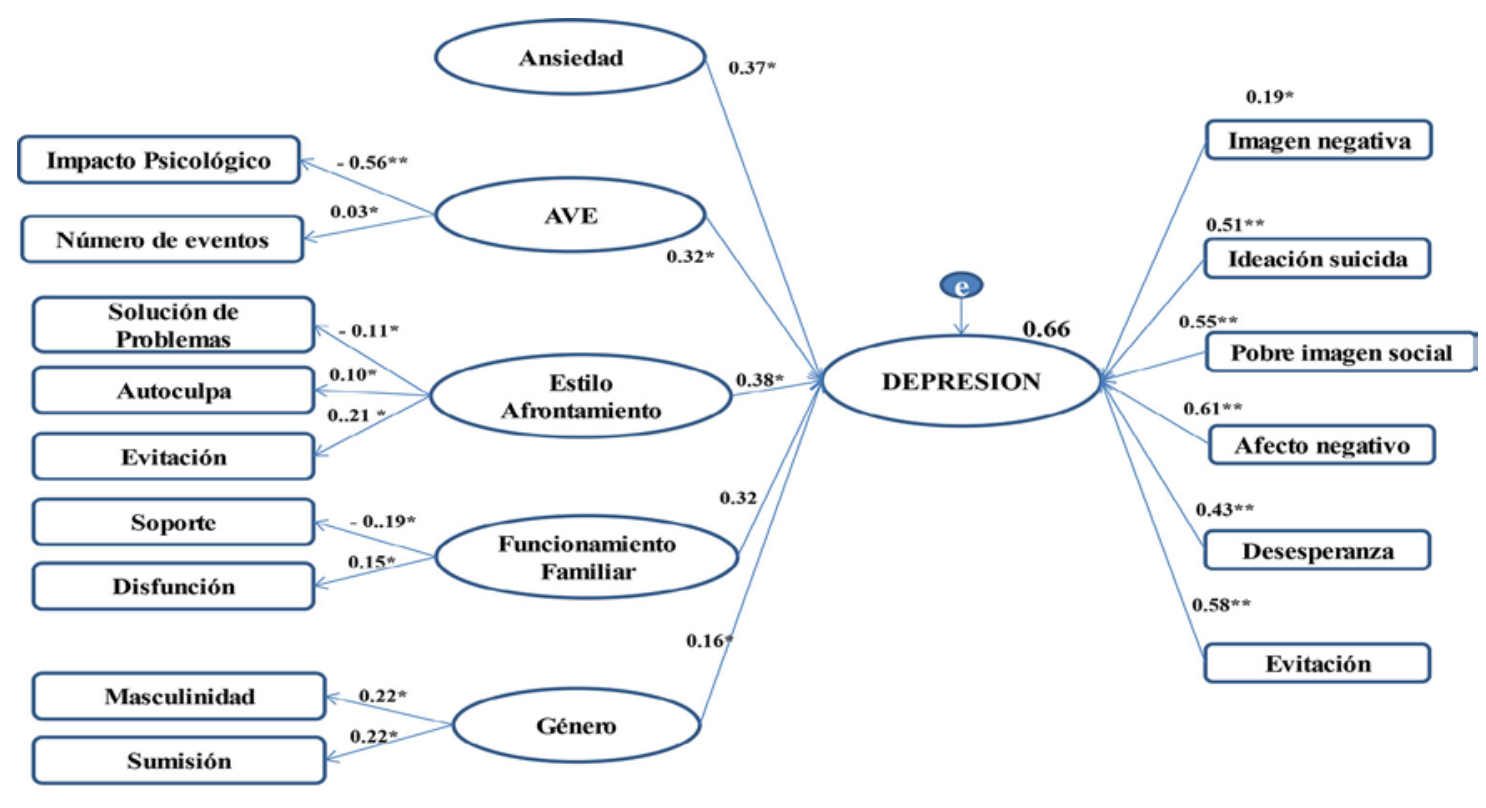

Notas: $* \mathrm{p} \leq 0,05 . * * \mathrm{p} \leq 0,01$.

Figura 1. Factores asociados a la severidad de la depresión en hombres representados en el Modelo de Ecuaciones Estructurales

aportando en la misma medida (PRVE=0.22). Todas las relaciones descritas del modelo cumplen el criterio mínimo de ser significativas con un $\mathrm{p} \leq 0.05$ (véase Figura 1).

Al calcular los índices corregidos para el tamaño de la muestra (Véase Tabla 5), se encontró que todos denotan que el modelo tiene un nivel de ajuste adecuado, ya que aunque el RSMEA no es inferior a 0.10 , el NFI sí es mayor a 0.80 , el CFI es superior a 0.95 y el $\mathrm{X}^{2}$ como índice de bondad del ajuste no fue significativo ya que el $p$ hallado era superior a 0.05 , es decir, que el modelo está identificado porque sus parámetros lo están; y que es recursivo porque no tiene efectos circulares o recíprocos entre sus variables.

Tabla 5.

Índices de ajuste del modelo de ecuaciones estructurales identificado de severidad de la depresión en hombres

\begin{tabular}{lccc}
\hline Índices de ajuste & & Resultado & Indicador \\
\hline Raíz cuadrada del error & RSMEA & 0.13 & - \\
Cuadrático medio & NFFI & 1.00 & - \\
Índice de ajuste normalizado & CFI & 1.00 & - \\
Índice comparativo de ajuste & X2 & 0.0 & $\mathrm{p}=0.097$ \\
Chi cuadrado & G1 & 2 & - \\
Grados de libertad & R2 & 0.59 & $\mathrm{p}=0.001$ \\
\hline
\end{tabular}

Notas: p: Nivel de significancia.

\section{DISCUSIÓN}

De acuerdo con los datos obtenidos, es posible afirmar que existe subreporte epidemiológico de casos de depresión en hombres en Colombia, probablemente debido al uso de instrumentos derivados del sistema DSM y CIE que no recogen de manera precisa los síntomas propios de la depresión masculina. Respecto de la prevalencia de corte en el último mes (agosto a noviembre), el 7.9\% de hombres encuestados padecían de depresión, proporción notablemente por encima de la reportada en estudios nacionales previos (6\% a 6.2\%). (FMSM, 2012; Gómez-Restrepo, 2004; MCPS, 2003).

El $6.1 \%$ de los hombres tenía depresión moderada y el $0.8 \%$ presentaba depresión severa ( 1 de cada 10 ), sin que sea posible especificar si se trataba de un episodio de depresión mayor o del trastorno, debido a que en este estudio se asumió como concepto global la existencia de depresión clínica en general y no la identificación específica de los trastornos del estado de ánimo.

Si se comparan las cifras de prevalencia, hay dos vías posibles de explicación de la diferencia. En primer lugar, esta podría deberse al incremento ocurrido en los años que han trascurrido luego del Estudio Nacional de Salud Mental en Colombia, o en los otros estudios más recientes; y en segundo lugar, a que probablemente los instrumentos usados para hacer los seguimientos epidemiológicos hasta ahora en el país sean menos sensibles para detectar depresión 
en hombres. Tal como concluyen Chang et al. (2008), los resultados epidemiológicos disponibles son variables y dependen mucho del instrumento utilizado para medir, pues varían los aspectos de la depresión que son privilegiados y su nivel de sensibilidad. Y no se puede perder de vista que varios trabajos previos con medidas tomadas con dos instrumentos simultáneamente han permitido identificar que el Zung y el BDI en sus dos versiones son menos sensibles a detectar casos de depresión en hombres (Álvarez y Londoño, 2012; Galicia, Sánchez y Robles, 2009).

El 7.7\% de encuestados presentó alto riesgo suicida asociado a depresión severa, y el $12.2 \%$ reportó ideas de muerte o deseos de morir, asociadas a depresión moderada en la mayoría de los casos, pero no de forma exclusiva, ya que existe cerca del $4 \%$ de casos en los que desconoce la fuente de los deseos de morir. Los datos nacionales disponibles sobre el riesgo suicida indican que más del $6 \%$ de la población está en riesgo (FMSN, 2012), datos no concordantes con los resultados de este trabajo, pues aquí se halló un riesgo suicida notablemente mayor, incluso próximo al $20 \%$. Adicionalmente, ya se ha establecido con suficiente certeza que los hombres tienen mayor riesgo suicida, pues la mayor parte de los suicidios efectivos registrados en Colombia y el mundo son cometidos por hombres (FMSN, 2012; Haeffel et al., 2007; INMFL, 2013; Merikangas et al., 2011).

Aunque no puede equipararse ideación suicida y depresión, pues son variados los trastornos psicológicos y físicos que pueden llevar a alguien a considerar la posibilidad de quitarse la vida (enfermedad mental severa, condiciones de salud física que implican dolor, problemas económicos y motivos culturales), sí se ha establecido que al menos en la mitad de los casos el acto suicida está relacionado con diagnósticos previos de trastornos del estado de ánimo, en especial de depresión mayor (Arsenault-Lapierre, Kim y Turecki, 2004) y que los hombres tienen mayor riesgo que las mujeres de cometer efectivamente el acto suicida (OMS, 2012).

Si se retoma el hecho de que los hombres con depresión moderada también refirieron el deseo de quitarse la vida, aunque no siempre las ideas de muerte o la ideación conlleva al acto suicida efectivo, esta condición debe ser considerada como una señal de alarma pues su aparición ha sido relacionada por varios autores con el posterior incremento en la severidad de los síntomas y la consolidación de las ideas de quitarse la vida (Egan, Wade y Shafran, 2011; O'connor et al., 2012; Rasmussen, Elliot y O'Connor, 2012). En especial, si se tiene en cuenta que el contexto colombiano es rico en ambientes violentos y de desigualdad, que resulta propicio para reafirmar las creencias desesperanzadoras sobre la vida.
Así mismo, cerca del 11\% de los hombres presentó síntomas leves de depresión que indican la existencia de condiciones subclínicas que merecen atención y el desarrollo de acciones preventivas dirigidas a la población masculina, para sensibilizar sobre los síntomas, la importancia del problema y su posible impacto.

Además, no se puede perder de vista que el $10.9 \%$ de los encuestados presentaban un nivel subclínico de depresión; y esta condición puede agudizarse con relativa facilidad en un país como Colombia, en el que hay inestabilidad laboral, un sistema de salud pobre e ineficiente, sumado a la situación de conflicto armado de larga data. Condiciones que proveen a diario estrés moderado pero crónico, que ya ha sido identificadas como depresógenas por un grupo extenso de autores (Almeida et al., 2011; Sterner y Kalynchu, 2010; Vargas-Navarrio, Latorre-López y Parra-Cárdenas, 2011; Zou et al., 2012), ya que aumentan la reactividad, deterioran la capacidad real de respuesta y afectan la evaluación que la persona hace acerca de su capacidad.

Por otra parte, y aunque se esperaría que en un estudio de corte nacional se hiciera una comparación de prevalencias por departamentos, en la presente investigación esta comparación no fue posible debido a que la estrategia de recolección de los datos no posibilitó el contar con muestras representativas de cada departamento.

Los factores asociados a depresión en hombres, detectados en este y otros estudios son los EVE y el mal funcionamiento familiar. Estar expuesto a un alto número de estresores que ocurren de forma consecutiva (con un impacto psicológico moderado), no contar con adecuado soporte familiar y estar expuesto a un ambiente familiar disfuncional, incrementan el riesgo de padecer de depresión y que esta se haga más severa, como lo muestran hallazgos similares de estudios previos realizados por Folkman (2001). Más que el impacto psicológico de un evento estresante en particular, es la acumulación de eventos de impacto psicológico moderado lo que se asocia a la aparición y agudización de la depresión en hombres (Bogdan et al., 2013; Sterner y Kalynchu, 2010; Zou et al., 2012).

También se había hecho notable el efecto del funcionamiento familiar, pero se había planteado que era el alto soporte familiar percibido el que se asociaba a la depresión en los hombres. Sin embargo, al igual que en el presente estudio, que apunta en sentido contrario a este respecto, existe amplia evidencia del efecto negativo que tiene la falta de apoyo social, especialmente del que ofrece o se espera que ofrezca la familia (Dokin et al., 2013; Grav, Hellzen, Romild y Stordal, 2012; Pawlby et al., 2009), que combinado con alto estrés disminuyen la capacidad de respuesta ante tal estrés. 
El pobre soporte familiar reportado por los hombres deprimidos tiene dos posibles explicaciones. En la primera, se aduce que el grupo familiar no cuenta con las habilidades para brindar apoyo debido a la dinámica familiar (Drydakis, 2013; Vallejo Casarín et al., 2008); y en la segunda, se plantea que aunque es capaz de hacerlo, no lo lleva a cabo debido a que el hombre no lo solicita para no ir en contra de los preceptos sociales (Burton, Stice y Seeley, 2004; Galea et al., 2007). Los resultados de este estudio indican que es precisamente la combinación de un ambiente familiar empobrecido y disfuncional que no provee apoyo, y la tendencia masculina a evitar pedir ayuda, la que está asociada con el reporte de depresión en los hombres.

Alto estrés, con bajo soporte y un estilo de afrontamiento dirigido principalmente a aliviar la emoción, poco centrado en encontrar salida a la situación problemática y estresante en el que predomina la evitación, y la tendencia a no actuar frente a los problemas (baja solución de problemas), además de la continua sensación de culpabilidad (alta autoculpa), son factores que facilitan la depresión, pues no le permiten al hombre ajustarse plenamente a la situación, ya que le impiden comprenderla y por ende solucionarla, (Carver y Connor-Smith, 2010; Gómez y Aclaren, 2006; Hernángomez Criado, 2012). También es conocido que el impacto de los eventos depende de la valoración que cada uno hace acerca de los recursos de que dispone para afrontar cada evento, y en este caso es sin duda negativa, pues está basada en la baja capacidad percibida observada en los hombres deprimidos (Barcelata et al., 2011; Barrett y Turner, 2005).

El papel de la ansiedad se incrementa en especial si se da simultáneamente con una expresión de género que combina alta sumisión/alta masculinidad.

Tanto la ansiedad como la masculinidad/feminidad fueron incorporadas al modelo obtenido, y se encontró que están asociadas a la severidad de la depresión en hombres como se había visto en otros estudios (Campos y Martínez Larrea, 2002; Conner et al., 2008; Merikangas, 2009). Aunque la combinación inesperada de alta sumisión/alta masculinidad no había sido vista, ya que indica la coexistencia de dos características casi opuestas como la sumisión y la masculinidad, esa condición es difícil de asimilar, tanto a nivel teórico como a nivel social, pues resulta incomprensible que esté presente en una misma persona, dado que implica pensar en alguien con alta dominancia e independiente, pero igualmente dependiente y sumiso. A este respecto se debe ahondar en próximos estudios y reconfirmar si realmente existe dicha ambivalencia psicológica, que de por sí ya haría comprensible el hecho de que quien vive esta disonancia sufra y se desajuste emocionalmente.

Si se quiere explicar esta combinación inusual, podría pensarse que los resultados apuntan a mostrar alta ambiva- lencia entre lo que se es y lo que se desea ser; es decir, que el hombre desea ser realmente independiente y dominante, y así se proyecta socialmente, pero en la intimidad realmente se percibe como alguien imperfecto por ser indeciso, necesitado de apoyo y comprensión, cansado de asumir el reto social de ser independiente, protector y fuerte.

La sumisión es el extremo opuesto de lo que se considera masculino; así, en los estándares sociales impuestos para los hombres se incluyen cuatro condiciones: posesión, fortaleza, capacidad y autoridad. Las tres primeras están relacionadas con la productividad (Broom y Trovey, 2009), y la última es opuesta a la sumisión. Entonces se espera que el hombre "masculino" sea altamente productivo y con capacidad económica, condiciones que le dan estatus y reconocimiento social; por ende, no ser productivo o no tener autoridad es símbolo de debilidad y de "falta de hombría".

Por el contrario, como lo hizo evidente el estudio de Adinkrah (2012), los grupos sociales altamente machistas esperan de los hombres agresividad, dominancia y arrojo; $y$ vetan las manifestaciones públicas de emociones que indican "debilidad", como miedo, tristeza o dolor, que expresen dependencia o que indican dependencia o subordinación.

La inflexibilidad del rol provoca su expresión negativa, pues se busca alcanzar (incluso de forma extrema) las características masculinas, aún por encima del propio bienestar. Aunque actualmente y con los cambios sociales se acepta que los hombres puedan poseer ciertas características tradicionalmente adscritas a la feminidad, la idea de que nunca deben ser sumisos aún se mantiene. Estos conceptos derivados del rol a cumplir se comportan como esquemas disfuncionales y los hombres los utilizan de manera automática para evaluar el mundo y el propio comportamiento ante los eventos estresantes (Beck, 2008).

En suma, los hombres forman parte de una cultura que les exige perfección, productividad, autonomía, valor y estabilidad emocional; están rodeados de un entorno rico en estresores que impactan moderada pero constantemente su funcionamiento emocional; viven en un grupo familiar que deben proteger y sostener, pero que les brinda pobre soporte y un ambiente caracterizado por los problemas de comunicación (críticas, juicios y continuas discusiones). Todo esto genera, en conjunto, el empobrecimiento de la imagen de sí mismos, la disminución de la autoeficacia y la frustración, lo cual les puede hacer sentir sin opciones, con sensación de vacío, pero al mismo tiempo retados a seguir adelante evitando las sensaciones que la misma cultura evitó que aprendieran a reconocer y a vivir de forma más positiva.

Aunque se haya logrado realizar un estudio con la más alta participación de hombres hasta ahora efectuada en Colombia, la consecución de la muestra fue una tarea compleja, ya que a pesar del apoyo decidido del equipo de 
trabajo, su dedicación y profesionalismo en la aplicación estricta del protocolo de contacto y recolección de los datos, un buen número de hombres se negó a contestar los cuestionarios, los diligenció sin firmar el consentimiento informado o dio respuestas al azar.

También es necesario estudiar la comorbilidad con otros trastornos y los límites reales de la expresión de cada uno de ellos, para determinar si los hallazgos son debidos a la coocurrencia de trastornos y no a que la depresión en hombres realmente se diferencia de la expresada por las mujeres.

\section{REFERENCIAS}

Adam, E. K., Doane, L. D., Zinbarg, R. E., Mineka, S., Craske, M. G. y Griffith, J. W. (2010). Prospective prediction of major depressive disorder from cortisol awakening responses in adolescence. Psychoneuroendocrinology, 35, 921-931. Doi:10.1016/j.paid.2011.02.008

Adinkrah, M. (2012). Better dead tan dishonored: Masculinity and male suicidal behavior in contemporary Ghana. Social Science y Medicine, 74(4), 474-481. Doi: 10.1016/j.socssimed.2010.10.011.

Almeida, O., Alfonso, H., Pirkis, J., Kerse, N., Sim, M., Flicker, L., Snowdon, J., Draper, B., Byrne, G., Goldney, R., Lautenschlager, N., Stocks, N., Scazufca, M., Huisman, M., Araya, R. y Pfaff, J. (2011). A practical approach to assess depression risk and to guide risk reduction strategies in later life. International Psychogeriatrics, 23(2), 280-291. Doi: $10.1017 / \mathrm{S} 1041610210001870$

Almeida, O., Pirkis, J., Kerse, N., Sim, M., Flicker, L., Snowdon, F., Draper, B., Byrne, G., Lautenschlager, N., Stocks, N., Alfonso, H. y Pfaff, J. (2012). Socioeconomic disadvantage increases risk of prevalent and persistent depression in later life. Journal of Affective Disorders, 138(3), 322-331. Doi: 10.1016/j.jad.2012.01.021.

Álvarez, N. y Londoño, C. (2012). Diseño y validación del Cuestionario de Depresión en Hombres. Tesis de Maestría. Universidad Católica de Colombia.

American Psycological Asociation. (2013). Diagnostic and statistical manual of mental disorders, DSM- $V$. Washington, DC: American Psychiatric Association.

Arsenault-Lapierre, G., Kim, C. y Turecki, G. (2004). Psychiatric diagnoses in 3275 suicides: a meta-analysis. BCM Psychiatry, 4, 37-47. Doi:10.1016/j.jmhg.2005.05.004

Barcelata, B., Durán, E. y Gómez-Maqueo, L. (2012). Valoración subjetiva de los sucesos de vida estresantes en dos grupos de adolescentes de zonas marginadas. Salud Mental, 35(6) 513-520. Recuperado de http://www.scielo.org.mx/ scielo. php?pid=S0185-3252012000600009yscript $=$ sci_ar ttext

Barrett, A. y Turner R. (2005). Family structure and mental health: The mediating effects of socioeconomic status, family process, and social stress. Journal of Health Social Behavior, 46(2), 156-169. Recuperado de http://www.scielo. org.mx/scielo.phpscript $=$ sci nlinksyref $=2465105 \mathrm{ypid}=\mathrm{S} 0185$ 3325201200060000900020 ylng=es

Beck, A. T. (2008). The evolution of the cognitive model of depression and its neurobiological correlates. American Journal of Psychiatry, 165(8), 969-977. Doi: 10.1176/appi. ajp.2008.08050721

Bogdan, R., Nikolova, Y. y Pizzagalli, D. (2013). Neurogenetics of depression: A focus on reward processing and stress sensitivity. Neurobiology of Disease, 52, 12-23. Doi:10.1016/j. nbd.2012.05.007

Broom, A. y Tovey, P. (2009). Men's Health. United Kingdom: Wiley.

Burton, E., Stice, E. y Seeley, J. R. (2004). A prospective test of the stress-buffering model of depression in adolescent girls: No support once again. Journal of Consulting and Clinical Psychology, 72, 689-697. Doi: 10.1097/00004583200001000-00016.

Caballo, V. (2006). Manual para la evaluación clínica de los trastornos psicológicos. Trastornos de la edad adulta e informes psicológicos. Madrid: Pirámide.

Campos, M. S. y Martínez-Larrea, J. A. (2002). Trastornos afectivos: análisis de su comorbilidad en los trastornos psiquiátricos más frecuentes. Anales, 25(3), 117-136. Recuperado de http:// www.cfnavarra.es/salud/anales/textos/suple25_3.html

Chang, S. M., Hahm, B-J., Lee, Y-J., Shin, M. S., Jeon, H. J., Hong, J. P. y Cho, H. (2008). Cross-national difference in the prevalence of depression caused by the diagnostic threshold. Journal of Affective Disorders, 106(1-2), 159-167. Doi:10.1016/j.jad.2007.07.023.

Carver, C. S. y Connor-Smith, J. (2010). Personality and Coping. Annual Review of Psychology, 61, 679-704. Doi: 10.1146/ annurev.psych.093008.100352

Caspi, A., Hariri, A. R., Holmes, A., Uher, R. y Moffitt, T. E. (2010). Genetic sensitivity to the environment: the case of the serotonin transporter gene and its implications for studying complex diseases and traits. American Journal of Psychiatry, 167, 509-527. Doi: B300B285D519C934EB192E145997CA2B.zQKnzAySRvJOZcdfIziQ\%3a3

Conner, K., Pinquart, M. y Gamble, S. (2008). Meta-analysis of depression and substance use among individuals with alcohol use disorders. Journal of Substance Abuse Treatment, 37(2), 127-137. Doi: 10.1016/j.jsat.2008.11.007

Conradi, H. J., Ormel, J. y de Jonge, P. (2011). Presence of individual (residual) symptoms during depressive episodes and periods of remission: a 3 year prospective study. Psychological Medicine, 44, 1165-1174. Doi: 10.1017/S0033291710001911

Córdoba, J., Fuentes, A. yRuíz, C. (2011). Revisión Bibliográfica sobre características sociodemográficas y repercusiones de la depresión en el trabajador. Medicina y Seguridad en el Trabajo, 57(223), 174-187. Recuperado de http://scielo.isciii. es/pdf/mesetra/v57n223/revision.pdf

Dokin, R., Rubino, J., Allen, L., Friedman, J., Gara, M., Mark, M. y Menza, M. (2013). Predictors of treatment response to 
cognitive behavioral therapy for depression in Parkinson's disease. Journal of Consulting Clinical Psychology, 80(4), 694-699. Doi: 10.1037/a0027695

Drydakis, N. (2013). Mulling at school and lab our market outcomes. IZA Discussion Paper, 7432, mayo. Recuperado de http://ftp.iza.org/dp7432.pdf

Egan, S., Wade, T. y Shafran, R. (2011). Perfectionism as a trans diagnostic process: A clinical review. Clinical Psychology Review, 31, 203-212. Doi:10.1016/j.cpr.2010.04.009

Federación Mundial de Salud Mental. (2012). Depresión: Una crisis global. Día Mundial de la Salud Mental 2012. Recuperado de http://www.wfmh.org/2012DOCS/WMHDay\%20Packet\%20 -\%20\%20Spanish \%20Translation\%202.pdf

Friedman, E. S. y Thase, M. E. (1995). Trastornos del estado de ánimo. En V. Caballo, G. Buelay J. A. Carrobles (dirs.), Manual de Psicopatología y Trastornos Psiquiátricos, vol. 1 (pp. 619-681). Madrid: Siglo XXI.

Fellinger, J., Holzinger, D., Sattel, H., Laucht, M. y Goldberg, D. (2009). Correlates of mental health disorders among children with hearing impairments. Development Medical Child Neurology, 51(8), 635-641. Doi: 10.1111/j.1469-8749.2008.03218.

Folkman, S. (2001). Revised coping theory and the process of bereavement. En M. S. Stroebe y R. O. Hansson (Eds.), Handbook of Bereavement Research: Consequences, Coping and Care (pp. 563-584). Washington, DC: American Psychological Association.

Forero, L., Avendaño, M., Duarte, C. y Campo-Arias, A. (2006). Consistencia interna y análisis factorial del APGAR. Revista Colombiana de Psicología, 35(1), 23-29. Recuperado de http://www.scielo.org.co/scielo.php?script=sci_arttextypid $=\mathrm{S} 0034-74502006000100003$

Galea, S., Ahern, J., Nandi, E., Tracy, M., Beard, J. y Vlavov, D. (2007). Urban neighborhood poverty and the incidence of depression in a population-based cohort study. Annals of Epidemiology, 17(3), 171-179. Doi:10.1016/j.annepidem.2006.07.008.

Galicia, I., Sánchez, A. y Robles, P. J. (2009). Factores asociados a la depresión en adolescentes. Anales de Psicología, 25(2), 227-240. Doi:10.6018/87501

Gotlib, I. y Hammen, C. (2012). Handbook of Depression. Nueva York: Guilford Press.

Gobierno de Canarias. (2010). Antidepresivos en el tratamiento de la depresión. Bolcan, buen uso de los medicamentos. Canarias: España.

Gómez-Restrepo, C., Bohórquez, A., Pinto Masis, D., Gil Laverde, J. F. A., Rondón Sepúlveda, M. y Díaz Granados, N. (2004). Prevalencia de depresión y factores asociados con ella en la población colombiana. Revista Panamericana de Salud Pública, 16(6), 378-86. Doi:10.1590/S1020-49892004001200003

Grav, S., Hellzen, O, Romild, U. y Stordal, E. (2012). Association between social support and depression in the general population: The Hunt study, a cross-sectional survey. Journal of Clinical Nursing, 21(1-2), 111-120. Doi: 10.1111/j.13652702.2011.03868

Haeffel, G., Abramson, L., Brazy, P., Shah, J., Teachman, B. y Nosek, B. (2007). Explicit and implicit cognition: A preliminary test of a dual-process theory of cognitive vulnerability to depression. Behaviour Research and Therapy, 45(6), 11551167. Recuperado de http://projectimplicit.net/papers.html

Heponiemi, T., Elovainio, M., Kivimäki, M., Pulkki, l., Puttonen, S. y Keltikangas-Järvinen, L. (2006). The longitudinal effects of social support and hostility on depressive tendencies. Social Science y Medicine, 63(5), 1374-1382. Doi: 10.1016/j. socscimed.2006.03.036

Hernangómez Criado, L. (2012). Vulnerabilidad cognitiva a la depresión: relación entre sesgos atencionales, Tesis Doctoral, Universidad Complutense de Madrid. Recuperado de http:// eprints.ucm.es/16407/

Huurre, T., Eerola, M., Rahkonen, O. y Does, H. (2006). Social support affect the relationship between socioeconomic status and depression? A longitudinal study from adolescence to adulthood. Journal of Affective Disorders, 100(1-3), 55-64. Doi: 10.1016/j.jad.2006.09.019

Ibarra-Alcantar, M., Ortiz-Guzmán, J., Alvarado-Cruz, F., Graciano-Morales, H. y Jiménez-Genchi, A. (2010). Correlatos del maltrato físico en la infancia en mujeres adultas con trastorno distímico o depresión mayor. Salud Mental, 33(4), 317-324. Recuperado de http://www.scielo.org.mx/pdf/sm/ v33n4/v33n4a3.pdf

Instituto Nacional de Salud Mental. (2013). Depresión. Recuperado de http://www.nimh.nih.gov/health/publications/espanol/ depresion/depresion.pdf

Juárez, F. y Guerra, R. (2011). Características socioeconómicas y salud en personas pobres y desplazadas. Psicología: Teoria e Pesquisa, 27(4), 511-519.

Karg, K., Burmesister, M., Shedden, K., y Sen, S. (2011). The serotonin transporter promoter variant (5-HTTLPR), stress, and depression meta-analysis revisited: evidence of genetic moderation. Archive General of Psychiatry, 68 (5), 444-454. Doi: 10.1001/archgenpsychiatry.2010.189.

Lara, M. (1991). Masculinidad, feminidad y salud mental: Importancia de las características no deseables de los roles de género. Salud Mental, 14(1), 12-18. Recuperado de http:// psycnet.apa.org/index.cfm?fa $=$ search.displayRecordyUID $=$ 1993-85005-001

Lund, C., Breen, A., Flisher, A., Kakuma, R., Corrigall, J., Joska, J., Swartz, L. y Patel, V. (2010). Poverty and common mental disorders in low and middle income countries: A systematic review. Social Science y Medicine, 71(3), 517-528. Doi: 10.1016/j.socscimed.2010.04.027

Mejía Castillo, A. de J. (2011). Estrés ambiental e impacto de los factores ambientales en la escuela. Pampedia, 7, 3-18. Recuperado de http://www.uv.mx/pampedia/numeros/numero-7/ Estres-ambiental-e-impacto-de-los-factores-ambientalesen-la-escuela.pdf 
Merikangas, K. R., Jin, R., He, J-P., Kessler, R., Lee, S., Sampson, N., Viana, M. C. Andrade, L. H., Hi, Ch., Karam, E., Ladea, M., Medina-Mora, M. E., Ono, Y., Posada-Villa, J., Sagar, R., Wells, E. y Zarkov, Z. (2011). Prevalence and correlates of bipolar spectrum disorder in the World Mental Health Survey Initiative. Archive General of Psychiatry, 68(3), 241-251. Doi:10.1001/archgenpsychiatry.2011.12

Ministerio de Protección Social Colombia. (2003). Informe Estudio Nacional de Salud Mental. Bogotá D.C.: Gobierno Nacional. Recuperado de http://www.minproteccionsocial. gov.co/vbecontent/library/documents/DocNewsNo15133DocumentNo1981.PDF.

Mittendorfer-Rutz, W. E. (2006). Trends of youth suicide in Europe during the 1980's and 1990's. Gender Differences and Implications for Prevention, 3, 250-257. Doi:10.1016/j. jmhg.2006.02.006.

Moore, S. M. y Hadjiyannakis, K. (2002). The Social Environment and Depression: Focusing on Severe Life Stress. En I. Gotlib y C. Hammen, (2002). Handbook of Depression. (pp. 314-340). Nueva York: Guilford Press.

LIdeación suicida: Identifying outpatients with entrenched suicidal ideation. Following Hospitalization.

Oliva, A., Jiménez, J., Parra, Á. y Sánchez-Queija, I. (2008). Acontecimientos vitales estresantes, resiliencia y ajuste adolescente. Revista de Psicopatología y Psicología Clínica, 13(1), 53-62. Doi: 10.1007/s10826-012-9681

Organización Mundial de la Salud (2012). Gender and Mental Health. Ginebra. Recuperado de http://www.Who.int/gender/ henderandhealth.html

Organización Panamericana de la Salud. (2012). Día Mundial de la Salud Mental. Recuperado de http://www.paho.org/arg/index. php?option $=$ com_contentyview $=$ articleyid $=1047$ Itemid $=325$

Palomar, J. y Cienfuegos, Y. (2007). Pobreza y apoyo social: un estudio comparativo en tres niveles socioeconómicos. Revista Interamericana de Psicología, 41(2), 177-188. Recuperado de http//:www.revista.unam.mx/vol.6/.../nov_art111.pdf

Pawlby, S., Hay, D. F., Sharp, D., Waters, C. S. y O'Keane, V. (2009). Antenatal depression predicts depression in adolescent offspring: Prospective longitudinal communitybased study. Journal of Affective Disorders, 113, 236-243. PMID:18602698

Rafful, C., Medina-Mora, M. E., Borges, G., Benjet, C. y Orozco, R. (2012). Depression, gender and the treatment gap in Mexico. Journal of Affective Disorders, 138(35), 1-11. Doi: 10.1016/j.jad.2011.12.040
Rasmussen, S., Elliot, M. y O'Connor, R. C. (2012). Psychological distress and perfectionism in recent suicide attempters: The role of behavioural inhibition and activation. Personality and Individual Differences, 52(6), 680-685. Doi: 10.1016/j. paid.2011.12.011

Rueda, C. y Vélez-Botero, H. (2010). Características psicométrica de un cuestionario de estilos de afrontamiento. Tipica Boletín Escolar, 6 (2).

Sander, J. y McCarty, C. (2005). Youth depression in the family context: Familial risk factors and models of treatment. Clinical Child and Family Psychology Review, 8, 203-219. Doi: 10.1007/s10567-005-6666-3

Sandín, B. (2003). El estrés: Un análisis basado en el papel de los factores sociales. Revista Internacional de Psicología Clínica y de la Salud, 3, 141-157. Recuperado de http://www.aepc. es/ijchp/articulos_pdf/ijchp-65.pdf

Sterner, E. y Kalynchu, L. (2010). Behavioral and neurobiological consequences of prolonged glucocorticoid exposure in rats: Relevance to depression. Progress in Neuro-Psychopharmacology and Biological Psychiatry, 34(5), 777-790. Doi:10.1016/j. pnpbp.2010.03.005

Troister, T. yHolden, R. (2010). Comparing psychache, depression, and hopelessness in their associations with suicidality: A test of Shneidman's theory of suicide. Personality and Individual Differences, 49(7), 689-693. Doi: 10.1177/0748175612451744

Vallejo Casarín, A., Osorno Murguía, R. y Mazadiego Infante, T. (2008). Estilos parentales y sintomatología depresiva en una muestra de adolescentes veracruzanos. Enseñanza e Investigación en Psicología, 3(1), 91-105.

Vargas-Navarrio, P., Latorre-López, D. y Parra-Cárdenas, S. (2011). Estresores psicosociales y depresión mayor recurrente. Revista Salud Bosque, 1 (2), 39-53. Doi: 10.4067/ S0717-92272002000500005.

Vásquez, L. F., Muñoz, R. y Becoña, E. (2000). Depresión: diagnóstico, modelos teóricos y tratamiento a finales del siglo XX. Psicología Conductual, 8(3), 417-449. Recuperado de http://www.behavioralpsycho.com/PDFespanol/ 2000/ art02.3 -.08.pdf

Vrshek-Schallhorn, S., Doane, L. D., Mineka, S., Zinbarg, R. E., Craske, M. G. y Adam E. K. (2012). The cortisol awakening response predicts major depression: predictive stability over a 4-year follow-up and effect of depression history. Psychosomatic Medicine, 1-11. Doi:10.1017/S0033291712001213

Zou, Y. F., Wang, F., Feng, X., Li, W., Tao, J. H., Pan, F. M., Huang, F. y Su, H. (2012). Meta-analysis of FKBP5 gene polymorphisms association with treatment response in patients with mood disorders. Neuroscience letters, 484(1), 56-61. Doi: 10.1016/j.neulet.2010.08.019 